\title{
Evaluation of automated malaria diagnosis using the Sysmex XN-30 analyser in a clinical setting
}

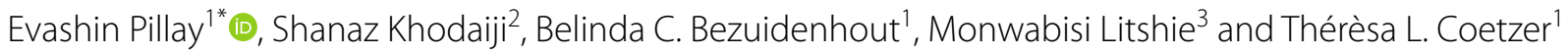

\begin{abstract}
Background: Early and accurate diagnosis of malaria is a critical aspect of efforts to control the disease, and several diagnostic tools are available. Microscopic assessment of a peripheral blood smear enables direct visualization of parasites in infected red blood cells and is the clinical diagnostic gold standard. However, it is subjective and requires a high level of skill. Numerous indirect detection methods are in use, but are not ideal since surrogate markers of infection are measured. This study describes the first clinical performance evaluation of the automated Sysmex XN-30 analyser, which utilizes fluorescence flow cytometry to directly detect and quantitate parasite-infected red blood cells.

Results: Residual EDTA blood samples from suspected malaria cases referred for routine diagnosis were analysed on the XN-30. Parasitaemia was reported as a percentage, as well as absolute numbers of infected red blood cells, and scattergrams provided a visual image of the parasitized red blood cell clusters. The results reported by the XN-30 correlated with microscopy and the analyser demonstrated $100 \%$ sensitivity and specificity. Measurements were reproducible and storage of samples at room temperature did not affect the parameters. Several Plasmodium species were detected, including Plasmodium falciparum, Plasmodium vivax and Plasmodium ovale. The XN-30 also identified the transmissible gametocytes as a separate cluster on the scattergrams. Abnormal red blood cell indices (low haemoglobin and raised reticulocyte counts), haemoglobinopathies and thrombocytopenia did not interfere with the detection of parasites. The XN-30 also generated a concurrent full blood count for each sample.

Conclusions: The novel technology of the Sysmex XN-30 provides a robust, rapid, automated and accurate platform for diagnosing malaria in a clinical setting. The objective enumeration of red blood cells infected with Plasmodium species makes it suitable for global use and allows monitoring of the parasite load once therapy has been initiated, thereby providing an early marker of drug resistance. The automated generation of a full blood count for each sample provides an opportunity for detecting unsuspected cases. Asymptomatic carriers can also be identified, which will be useful in blood transfusion centres, and will enable treatment of these individuals to prevent the spread of the disease.
\end{abstract}

Keywords: Plasmodium falciparum, Automated diagnosis, Sysmex XN-30 analyser

\footnotetext{
*Correspondence: evashinp@gmail.com

${ }^{1}$ Wits Research Institute for Malaria, Department of Molecular Medicine

and Haematology, School of Pathology, Faculty of Health Sciences,

University of the Witwatersrand and National Health Laboratory Service,

Johannesburg, South Africa

Full list of author information is available at the end of the article
} 


\section{Background}

\section{The global burden of disease}

Malaria remains one of the most important parasitic diseases globally, with more than 3.2 billion people in 91 countries at risk of infection [1]. The clinical and public health impact of the disease is geographically variable, depending on the intensity of transmission and the parasite species involved. The World Health Organization (WHO) 2018 malaria report indicates that worldwide in 2017, there were approximately 219 million cases with an estimated death toll of 435,000. Ninety-three percent of all deaths due to the disease occurred in sub-Saharan Africa, affecting mainly young children and pregnant women [1]. The report also shows that after an unprecedented period of success in global malaria control, the number of cases has increased, and progress has stalled. Of concern is that several countries which carry a disproportionate burden of disease have reported increases in malaria cases. Notably, the ten highest burden African countries documented an estimated 3.5 million more cases in 2017 compared to the previous year [1]. In this regard effort is needed to reverse this trend and every case of malaria prevented and death averted, contributes to the global drive towards elimination.

\section{The role of currently recommended malaria diagnostic tools}

To combat the burden of malaria effectively, early and accurate diagnosis is important. Not only will a correct diagnosis reliably distinguish malaria from other causes of acute febrile illness, but it will also permit specific treatment to be initiated thereby preventing complications and reducing mortality. Unfortunately, clinical diagnosis alone has a very low specificity and is not accurate enough, even with a travel history, as the presenting clinical symptoms and signs of malaria are often non-specific and can mimic other tropical infections [2]. According to the WHO, malaria diagnosis based solely on clinical features often leads to overtreatment and irrational use of anti-malarial agents [3].

Current diagnostic recommendation by the WHO is for all suspected malaria cases to have a parasitological test to avoid presumptive therapy and minimize unnecessary exposure to anti-malarial drugs. The two recommended methods for parasitological confirmation are microscopy for direct detection of the parasite, and immuno-chromatographic rapid diagnostic tests (RDT) for indirect detection based on the presence of malarial antigen or protein [3]. It is noteworthy that assays based on polymerase chain reaction (PCR) and loop-mediated isothermal amplification (LAMP) have demonstrated high sensitivity and specificity for malaria detection, and by comparison have proven that infections can be missed by microscopy and RDTs $[4,5]$. However, these methods are not a practical alternative for utilization in many malaria endemic regions because they are laborious and necessitate a high level of skill.

\section{Microscopy}

For more than a century microscopy has served as the preferred diagnostic test for malaria. Thorough review of a well-prepared and well-stained peripheral blood smear by a skilled microscopist remains the clinical gold standard for malaria parasite detection [6]. Not only does it represent a sensitive and highly specific method for diagnosis, but it also allows quantitation of malaria parasitaemia, species classification and identification of the different life cycle stages [3, 7]. In endemic areas, microscopy can also enable the detection of co-infections including other parasites e.g. babesiosis (a tick-borne parasitic disease that can be confused with malaria); filariasis (seen as motile microfilariae in thick and thin films); and haemoflagellates e.g. Trypanosoma and Leishmania species. Bacteria may also be detected e.g. in louse- and tick-borne relapsing fevers, spirochetes of Borrelia species can be observed between cells; in septicaemia, bacteria can be seen within neutrophils e.g. streptococci and staphylococci; and in bartonellosis, the causative organism can be appreciated on the surface of red blood cells with associated haemolytic anaemia and spherocytosis. In addition, fungi e.g. Candida albicans and Histoplasma capsulatum can be observed within neutrophils and monocytes, respectively [8]. However, microscopy is not without its challenges and the quality of results is highly variable, as demonstrated by external assessments recently conducted at selected facilities [9]. Accurate diagnosis depends on the quality of the stained peripheral blood smear, the availability and proper functioning of a microscope, the skill and experience of the microscopist, appropriate methods for validating proficiency, and ongoing supervision and training to maintain an acceptable level of expertise. Further challenges include subjective result interpretation, and a labourintensive and time-consuming test procedure. An automated microscopist would improve malaria diagnosis. To this end, Eshel and colleagues [10] recently evaluated the performance of a novel desktop instrument, the Parasight (Sight Diagnostics, USA), which uses digital fluorescence microscopy and computer vision algorithms for malaria diagnosis. The sensitivity and specificity for parasite detection when compared to PCR, ranged from 99.0-99.3\% and $98.9-100 \%$ in two study sites, in India and Kenya, respectively. The instrument was also able to identify Plasmodium falciparum and Plasmodium vivax species correctly in $100 \%$ of cases analysed in India, but in Kenya this decreased to $96.1 \%$ for P. falciparum cases. 


\section{Immuno-chromatographic RDTs}

The other recommended parasitological test that has gained interest in the last two to three decades is the RDT. This technology utilizes monoclonal antibodies on a test strip, which are directed against parasite-specific enzymes or antigens present in the blood of infected individuals [11]. These commercially available tests can detect different malaria species and are generally utilized where quality-assured microscopy is not available [3]. Although they may serve as a useful complement or alternative to microscopy, there are some noteworthy drawbacks. Firstly, they are not quantitative, thereby making them unsuitable for prognostic significance of infection or monitoring of parasite clearance as a measure of treatment efficacy. In addition, they do not distinguish a new infection from a recently treated one, since the malaria antigen can persist for up to 2 months post-treatment [12]. Moreover, they have limited shelf life and are sensitive to high temperatures [7]. They also generate false positive results following cross-reactivity with antigens such as rheumatoid factor [13]. Furthermore, histidinerich protein 2 (HRP2) gene deletions enable the $P$. falciparum parasite to avoid detection by HRP2-based RDTs, thereby generating false negative results [14]. Lastly, currently available RDTs were developed prior to the discovery that Plasmodium knowlesi can cause significant disease in humans and were, therefore, not adequately evaluated for detecting this species [15]. Infection with $P$. knowlesi may thus be missed in patients with a negative RDT if microscopy is not performed, with potentially fatal consequences.

\section{Malaria detection by automated analysers}

As the field of medical diagnostics continues to evolve, there is a constant search for alternative methods to detect and quantify malaria parasites. To reduce analytical time and improve accuracy, automation of the malaria diagnostic process is highly desirable. Automated haematology analysers can offer fast, sensitive and cost-effective assessment of all suspected malaria cases [16]. Several studies have identified unusual light scatter patterns generated during routine full blood count (FBC) analysis of malaria-infected blood samples [17-20]. It has been proposed that these atypical scattergrams are due to white blood cells (WBC), which scatter light differently after they have phagocytosed the parasite and contain birefringent haemozoin pigment $[17,21,22]$. Such evidence is diagnostically impressive; however, these findings only suggest the diagnosis and confirmation by microscopic assessment of a peripheral blood smear will still be mandatory [16]. Also, intra-erythrocytic haemozoin has been detected using specialized flow cytometry methods, however, it is not optimal for diagnosis of $P$. falciparum malaria since the circulating ring forms of the parasite contain minimal amounts of haemozoin [23].

Sysmex haematology analysers (Sysmex Corporation, Kobe, Japan) uniquely utilize the principle of fluorescence flow cytometry to count and differentiate blood cells. Using this technology, the corporation developed a prototype analyser, the $\mathrm{XN}-10(\mathrm{M})$, for the automated detection of malaria. Performance data from the prototype study were used to optimize the software algorithms, which led to the development of the $\mathrm{XN}-30$. This analyser is currently only available for research purposes, but regulatory approval is pending, and the analyser is scheduled for commercial launch in 2019.

A recent evaluation study of the XN-30 by Tougan and colleagues [24] using $P$. falciparum parasites cultured in vitro, demonstrated excellent correlation of automated parasitaemia with microscopy. However, the parasitaemia in healthy asexual $P$. falciparum cultures typically does not exceed $10 \%$, which is much lower than the parasite burden that may be present in severely ill malaria patients. In addition, asexual parasite cultures contain all the intra-erythrocytic developmental stages including ring forms, trophozoites and schizonts, but lack gametocytes, which are formed when a developmental switch to sexual reproduction occurs in selected infected red blood cells (RBC). Parasite cultures therefore differ from the clinical scenario where a blood sample from a patient with $P$. falciparum malaria contains only ring forms and occasionally gametocytes, thus necessitating the evaluation of the analyser in a clinical setting.

This article outlines the technology behind automated malaria detection by Sysmex analysers and report on the initial prototype studies and subsequent evaluation of the diagnostic performance of the $\mathrm{XN}-30$ using blood samples from patients with malaria.

\section{Methods}

The prototype, $\mathrm{XN}-10(\mathrm{M})$, and its successor, the $\mathrm{XN}-30$, utilize the same malaria detection and measurement principle.

\section{Sysmex malaria detection and measurement principle}

Using fluorescence flow cytometry, the $\mathrm{XN}-30$ detects and counts malaria-infected RBCs (MI-RBC) and WBCs. It contains a blue semiconductor laser with a $405 \mathrm{~nm}$ beam, making it different from conventional Sysmex haematology analysers, which have a red semiconductor laser with a $633 \mathrm{~nm}$ beam. Compared to red light, the wavelength of blue light is shorter, thereby permitting detection of smaller particles. In the process of malaria detection, a newly developed reagent (Lysercell M) partially permeabilizes the cell membrane of RBCs allowing 
entry of a reagent with fluorescent properties (Fluorocell $\mathrm{M})$, which stains parasite nucleic acids. When a sample is analysed, the RBC count is measured using a sheath flow direct current (DC) detection method and the corresponding malaria-infected $\mathrm{RBC}$ count (MI-RBC\#; reported as parasites $/ \mu \mathrm{L}$ ) is measured by fluorescence flow cytometry. The percentage of malaria-infected RBCs (MI-RBC\%) is calculated from the ratio of the different RBC counts (MI-RBC\# and RBC) obtained from the two methods. Similar to conventional Sysmex analysers, the $\mathrm{XN}-30$ also utilizes the sheath flow DC detection method to determine other indices including the haematocrit and platelet count, and a sodium lauryl sulphate (SLS) method for measuring haemoglobin.

\section{Sysmex malaria scattergrams}

A malaria (M) scattergram is generated for each sample and consists of side fluorescence light (SFL) intensity on the horizontal axis ( $\mathrm{x}$-axis) and forward scattered light (FSC) intensity on the vertical axis (y-axis; Fig. 1). By virtue of the larger size and higher nucleic acid content of WBCs compared to RBCs, they generate a cluster on the upper right corner of the $\mathrm{M}$ scattergram (turquoise dots; Fig. 1a). Non-infected RBCs, platelets and debris on the other hand feature on the left side of the M scattergram, along the vertical axis (blue dots; Fig. 1a). The amount of nucleic acid in a single malaria parasite is less than $1 / 100$ of a human WBC $[25,26]$ and by combining this property with the principle of staining parasite nucleic acids, MI-RBCs can be distinguished from WBCs, non-infected RBCs, platelets and debris (Fig. 1b, c).

The number of parasites in RBCs can also be discriminated by comparing the fluorescence intensity within a sample. The XN-30 can distinguish singly-infected RBCs from those containing two or more ring forms, since the amount of nucleic acid contained within an infected RBC is different, and this is represented on the M scattergram as separate clusters (Fig. 1b, c).

Plasmodium develop sequentially through ring forms, trophozoites and schizonts during the asexual life cycle while gametocytes are formed after a developmental trigger to sexual reproduction. Each developmental stage contains different amounts of total stainable nucleic acid. Additionally, MI-RBCs increase in size as the parasite matures from a ring form to a trophozoite and schizont. Conceptually, the intensity of both SFL and FSC will be unique for each of these developmental forms. Thus, the $\mathrm{XN}-30$ categorizes malaria infection within a sample by life cycle stages based on the SFL, reflecting the amount of nucleic acid, and the FSC, reflecting the size of MIRBCs (Fig. 1b, c). Specifically, each dot on the M scattergram represents the $x$ (SFL): $y$ (FSC) co-ordinates of each individual MI-RBC. The XN-30 software algorithm detects distinct clusters of MI-RBCs within a specified signal range then classifies the life cycle stages and depicts this prediction by assigning specific colours to the dots (Fig. 1b, c). This concept of differentiation is similar to the Adaptive Cluster Analysis System (ACAS), which is utilized by Sysmex X-Class and XN-series haematology analysers for WBC differentiation. In brief, in contrast to fixed gating, the software confirms that a cluster exists (identified by a centroid) and that in the case of ring forms and gametocytes, such clusters are separated by a trough, within a defined signal range, allowing for biological variation. The distinction between trophozoites and schizonts is less clear, as these stages form a continuum (based on size and nucleic acid content), and hence the cut-off for classification between these two stages is fixed. The primary intention of classifying such life cycle stages is not to provide a quantitative differentiation, but rather
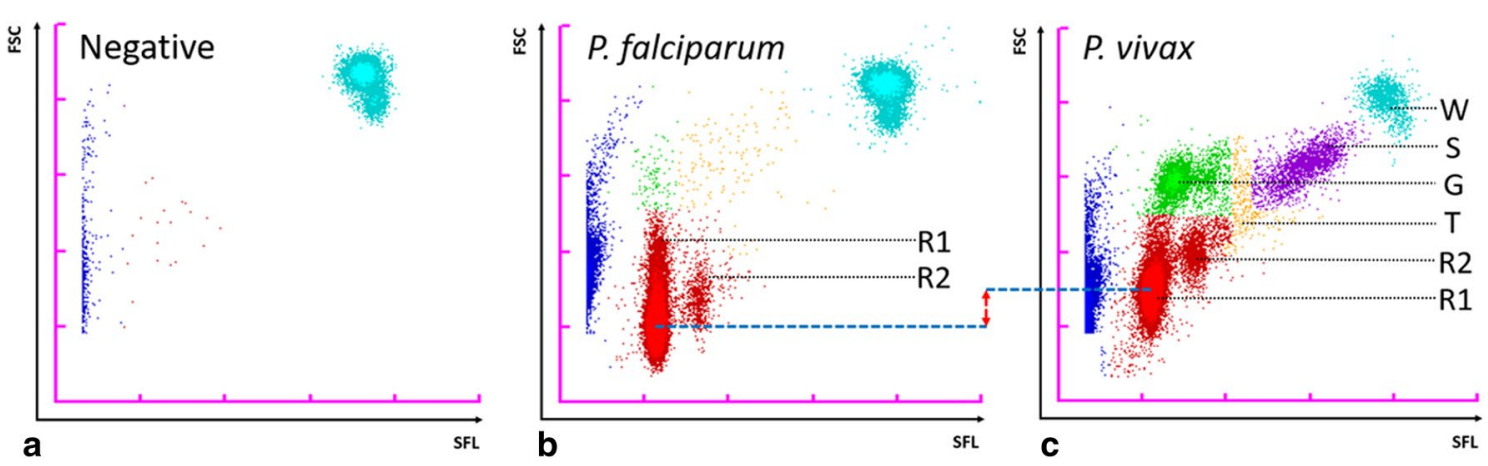

Fig. $1 \mathrm{M}$ scattergrams illustrating the principle of detection of red blood cells infected with malaria parasites. SFL: side fluorescence light; FSC: forward scattered light. Samples were analysed in WB mode. a Malaria-negative blood sample. Blue dots: non-infected RBCs, platelets and debris; red dots: background noise below the limit of quantitation. $\mathbf{b}$ RBCs infected with P. falciparum (MI-RBCs) showing R1: RBCs infected with 1 ring form, and R2: RBCs infected with 2 ring forms. c RBCs infected with P. vivax showing different parasite developmental stages. T: trophozoites; G: gametocytes; S: schizonts; W: white blood cells 
to show that developmental stages beyond ring forms are present in the peripheral circulation of an infected individual. Evidence of the latter, together with the size of MI-RBCs is used by the XN-30 to differentiate between species (Fig. 1b, c). The RBCs infected with $P$. falciparum contain small ring forms compared to $P$. vivax, which have different physical properties [27], thus generating distinct light scatter patterns. Furthermore, in clinical samples of $P$. falciparum infection, asexual ring forms are predominantly seen, in contrast to $P$. vivax where all the asexual stages are present (Fig. 1b, c).

\section{$\mathrm{XN}-30$ software improvements}

Based on the data analysis of the prototype study, changes were made to the software to improve the limit of quantitation, specificity, and to minimize interferences. Details of the software modification, including the algorithm flow, are provided in Additional file 1: Figs. S1 and S2.

\section{Study sites}

The concept of automated malaria parasite detection using the prototype $\mathrm{XN}-10(\mathrm{M})$ analyser was initially assessed in an urban clinical setting in Johannesburg, in the Republic of South Africa (RSA), from January to June 2014. A second study site, located in Mumbai, India, was added to enrich the data set with $P$. vivax cases from November 2014 to January 2015 and to evaluate the ability of the analyser to differentiate between Plasmodium species. The clinical evaluation of the diagnostic performance of the $\mathrm{XN}-30$ was conducted in RSA from January to June 2017.

\section{Patient samples}

In RSA, all adult patients presenting with clinical suspicion of malaria either to Chris Hani Baragwanath Academic Hospital or Charlotte Maxeke Johannesburg Academic Hospital were considered for inclusion. In India, only samples with a microscopic diagnosis of malaria, confirmed at the P. D. Hinduja National Hospital laboratory, were included. At both sites residual blood from specimens collected in EDTA tubes [Vacutainer ${ }^{\circledR}$ $\mathrm{K}_{2}$ EDTA $(1.8 \mathrm{mg} / \mathrm{mL})$, BD Bioscience, USA] and submitted to the local hospital laboratory for routine malaria diagnostic testing were utilized. To be eligible for the study, all samples were required to be at least $1 \mathrm{~mL}$ in volume and processed on the analyser by a dedicated operator within $24 \mathrm{~h}$ of collection from the patient. This study was approved by the Human Research Ethics Committee of the University of the Witwatersrand (M140995 and M160549) and by the National Health and Education Society (872-14-SK/KS), in RSA and India respectively.

\section{Evaluation of malaria parasites Sysmex analysers}

Samples were analysed on the prototype, $\mathrm{XN}-10(\mathrm{M})$, and the XN-30 analysers in whole blood (WB) mode. In addition, samples were processed in low malaria (LM) and pre-dilute $(\mathrm{PD})$ modes on the $\mathrm{XN}-30$. In the $\mathrm{LM}$ mode the volume of each sample measured is triple $(180 \mu \mathrm{L})$ that of the WB mode $(60 \mu \mathrm{L})$, to obtain improved sensitivity. In contrast, the PD mode requires only $20 \mu \mathrm{L}$ of WB sample diluted in $120 \mu \mathrm{L}$ of a standard diluent (Cellpack DCL), to enable analysis of small blood volume samples such as in elderly and paediatric patients. The $\mathrm{XN}-30$ is equipped to perform automatic conversion of results using the appropriate dilution factor.

Both analysers generate results automatically, which are presented as malaria-negative or malaria-positive, accompanied by a conventional FBC, MI-RBC\#, MI$\mathrm{RBC} \%$, an $\mathrm{M}$ scattergram and a flag with a suggested species classification, either as suspected $P$. falciparum or suspected 'others' (i.e. non-falciparum species).

\section{Peripheral blood smear microscopy}

For the prototype study, thin peripheral blood smears were prepared and evaluated by routine laboratory staff according to standard procedures [28]. For the XN-30 study, the microscopically confirmed parasite density of all malaria-positive samples was correlated with that determined by the analyser to evaluate its performance. For each sample referred for suspected malaria, two thin peripheral blood smears were prepared and stained using the RapiDiff stain kit (Clinical Sciences Diagnostics CC, RSA). All slides were examined by two expert microscopists using the Olympus BX41TF light microscope (Olympus Corporation, Tokyo, Japan) at 500 $\times$ magnification. The density of malaria parasites was determined according to the current WHO guidelines [28]. Briefly, percentage parasitaemia was calculated after counting the number of MI-RBCs per 10,000 RBCs. If parasites were not visualized after counting 10,000 RBCs, the sample was deemed to be microscopy negative.

\section{Immuno-chromatographic RDTs}

All samples were tested for the presence of malarial antigens and results recorded as either positive or negative. For the prototype study, the MAKROmed malaria rapid test kit (Makromed Manufacturing (Pty) Ltd, RSA) which detects the $P$. falciparum-specific HRP2 antigen, and the SureTest MAL malaria antigen test kit (pLDH VIVAX + HRP-II FAL; MicroGene Diagnostic Systems (P) Ltd, India), which detects both $P$. falciparum and $P$. vivax species were used. For the XN-30 study, the ICT 
Malaria dual test kit (ICT diagnostics, RSA), which detects $P$. falciparum, $P$. vivax, Plasmodium malariae and Plasmodium ovale antigens, was employed.

\section{Polymerase chain reaction}

In RSA, any discordant results between the analysers and other methods (microscopy and/or RDT) were resolved with qualitative PCR assays using Plasmodium-specific primers according to a standardized protocol adapted from Hang et al. [29]. For the XN-30 study, the PCR testing was conducted at the National Institute for Communicable Diseases (NICD) in Johannesburg.

\section{Interference by non-malarial factors}

To exclude potential interference by non-malarial factors, several samples, confirmed by microscopy and RDT to be malaria-negative, were processed in WB mode on both analysers and additionally in LM mode on the XN-30. These comprised random routine specimens with isolated low haemoglobin $<10 \mathrm{~g} / \mathrm{dL}$, isolated low platelet count $<100 \times 10^{9} / \mathrm{L}$ and raised reticulocyte counts $>5 \%$ or equivalent to $>100,000 \times 10^{6} / \mathrm{L}$. Additionally in the prototype study, samples from patients with HIV infection (RSA), confirmed acute Dengue infection and an eosinophil count $>1 \times 10^{9} / \mathrm{L}$ (India) were included. For the performance evaluation of the $\mathrm{XN}-30$, samples from patients with known haemoglobinopathies (thalassaemia and sickle cell disease) were also analysed.

\section{Analytical evaluation of the Sysmex XN-30}

The $\mathrm{XN}-30$ was assessed for the following additional performance parameters: limit of blank (LoB); limits of detection (LoD) and quantitation (LoQ); carryover; precision and stability.

\section{Limit of blank}

To determine the LoB, samples confirmed by microscopy and RDT to be malaria-negative, were processed. These comprised random routine specimens with normal FBC results. The LoB was calculated using the formula $\mathrm{LoB}=$ Mean $_{\text {Blank }}+1.645\left(\mathrm{SD}_{\text {Blank }}\right)[30]$.

\section{Limits of detection and quantitation}

To determine the LoD and LoQ, malaria-positive samples, confirmed by microscopy and RDT, were analysed and the MI-RBC\# confirmed. Each sample was also typed for $\mathrm{ABO}$ blood grouping using a standard kit (Rapid Labs, UK) and then diluted using ABO-compatible malaria-negative blood obtained from volunteers in the RSA research laboratory. Standards comprising various concentrations of parasites were generated for determining the LoQ (WB: 30 parasites/ $\mu \mathrm{L}$; LM: 20 parasites/ $\mu \mathrm{L}$; PD: 40 parasites/ $\mu \mathrm{L})$ and $\operatorname{LoD}(10,20,30,40,60$ and 100 parasites $/ \mu \mathrm{L}$ for each mode). Each standard was analysed ten times consecutively and the LoD was calculated using the formula $\mathrm{LoD}=\mathrm{LoB}+1.645$ ( $\mathrm{SD}_{\mathrm{Low}}$ concentration sample) [30].

\section{Carryover}

Carryover from a malaria-positive sample with high parasite density ( $>5 \%$ parasitaemia) to a malaria-negative sample was assessed by analysing the positive sample three times consecutively followed by three consecutive analyses of the negative sample. These steps were repeated using Cellpack DCL as a blank instead of the negative sample. Carryover was judged to be clinically significant if the first negative sample or the blank sample demonstrated a MI-RBC\# result $>$ LoQ.

\section{Precision}

To assess for precision, malaria-positive samples, confirmed by microscopy and RDT, and representing high $(>5 \%)$, medium $(1-2 \%)$ and low $(<1 \%)$ parasite densities were selected, each of which was analysed ten times consecutively.

\section{Stability}

To assess the impact of sample age on malaria classification, malaria-positive samples, confirmed by microscopy and RDT, were initially analysed within $24 \mathrm{~h}$ of collection from the patient. Each sample was divided into two aliquots, which were stored at $4-8{ }^{\circ} \mathrm{C}$ and room temperature $\left(20-25{ }^{\circ} \mathrm{C}\right)$, respectively, and subsequently analysed after approximately 48,72 and 96 h post sample collection. If there was insufficient sample volume to test both temperatures, preference was given to samples stored at room temperature.

\section{Purification and detection of gametocytes by the Sysmex XN-30}

Gametocytes from NF54 P. falciparum parasites were induced in vitro and purified as previously reported [31]. Mature stage V gametocytes were isolated after 14 days in culture using NycoPrep ${ }^{\mathrm{TM}}$ (Axis-Shield, Norway) density gradient centrifugation and magnetic separation on LS columns in a MidiMACS magnetic system (Miltenyi Biotec, Germany). Gametocytaemia was assessed in a Neubauer chamber. To evaluate gametocyte detection by the $\mathrm{XN}-30$, malaria-negative WB samples $(1 \mathrm{~mL})$ were spiked with increasing volumes of purified gametocytes (1 500 stage $\mathrm{V}$ gametocytes $/ \mu \mathrm{L}$ ) and analysed in $\mathrm{WB}$ mode. 


\section{Statistical analysis}

Correlation between parasitaemia measured by the $\mathrm{XN}-30$ and microscopy was analysed using regression analysis. The coefficient of determination $\left(R^{2}\right)$, means, standard deviations (SD), and coefficients of variation $(\mathrm{CV} \%=\mathrm{SD} \div$ mean $\times 100)$ were calculated using Analyse-it for Microsoft Excel version 5.01 (Microsoft, Redmond, WA, USA). Confidence intervals (CI; 95\%) for the sensitivity and specificity data were also determined using this software.

\section{Results}

\section{Evaluation of the prototype $\mathrm{XN}-10$ (M)}

A total of 1028 samples processed on the prototype analyser gave valid results for further analysis. There were 261 confirmed malaria-positive samples ( $P$. falciparum: $\mathrm{n}=157 ; P$. vivax: $\mathrm{n}=104)$, 261 confirmed malaria-negative samples from patients with an acute febrile illness in whom malaria had been suspected, 278 malaria-negative interference samples, 128 samples with normal FBC values and a further 100 randomly selected FBC samples from the routine laboratory in India.

The malaria detection of the $\mathrm{XN}-10(\mathrm{M})$ analyser was evidenced by a sensitivity of $97.7 \%$ ( $95 \%$ confidence interval $95.0-98.9 \%)$ and a specificity of $98.7 \%$ (95\% confidence interval 97.5-99.3\%). In eleven smear-negative cases the $\mathrm{XN}-10(\mathrm{M})$ detected malaria, all of which were subsequently confirmed by PCR to be true positive results. Furthermore, there was one "isolated low platelet count" interference sample that appeared to have given a false positive result on the $\mathrm{XN}-10(\mathrm{M})$ but was subsequently confirmed on smear review to be an unsuspected case of true malaria. All other interference samples, as well as all normal samples with interpretable results (refer to next paragraph), were correctly identified as malarianegative. There was no difference in malaria detection performance based on Plasmodium species. The analyser correctly identified the species as $P$. falciparum in $98 \%$ of cases (95\% confidence interval 94.2-99.3\%) and nonfalciparum in $94.8 \%$ of cases ( $95 \%$ confidence interval 88.4-97.8\%).

The biggest shortcoming observed was the high percentage $(14.8 \%)$ of indeterminate results. If the particle count in the "M" channel exceeded the lower LoQ, but no distinct cluster was observed in the malaria gating area, then the analyser generated an "abnormal MI-RBC scattergram" flag and did not issue a report on the presence of malaria. Indeterminate results were largely associated with malaria-negative samples $(19.4 \%$; 149/767), with only three of the malaria-positive cases $(1.15 \% ; 3 / 261)$ being affected. Based on these first performance data, the software was improved, resulting in the $\mathrm{XN}-30$ analyser.

\section{Evaluation of the Sysmex XN-30}

\section{Detection of malaria parasites}

A total of 191 samples were processed on the XN-30: 124 were confirmed to be malaria-positive (P. falciparum: $\mathrm{n}=122 ; P$. ovale: $\mathrm{n}=2$ ) and 67 were confirmed to be malaria-negative by microscopy and RDT, and where necessary also PCR $(n=5)$. Figure 2 provides examples of M scattergrams of two P. falciparum cases. Examples of RBC flags indicating the malaria species as P. falciparum 'P. $\mathrm{f}$ ' or 'others' are provided in Additional file 1: Fig. S3. The datasets generated and analysed in this study are available in Additional file 2: Tables S1 and S2. Some of the data have been published as an abstract [32].

\section{Comparison of parasitaemia between $\mathrm{XN}-30$ analysis and microscopy}

To validate the analyser results, parasitaemia determined by the $\mathrm{XN}-30$ was compared to clinical gold standard expert microscopic evaluation. A correlation analysis revealed a strong coefficient of determination with $\mathrm{R}^{2}=0.990$ for WB mode (Fig. 3). Analysis of samples in LM and PD modes revealed $R^{2}$ values of 0.991 and 0.986 , respectively (Additional file 1: Fig. S4). Similar findings were demonstrated by other groups using in vitro $P$. falciparum cultures $[24,33]$, and suggest that the $\mathrm{XN}-30$ is non-inferior to expert microscopy for the automated quantitation of malaria parasite density.

\section{Sensitivity and specificity}

The XN-30 demonstrated a sensitivity of $100 \%$ (95\% confidence interval $97.0-100 \%$ ) and a specificity of $100 \%$ (95\% confidence interval $92.6-100 \%$ ) for malaria parasite detection.

\section{Species identification}

The LM mode was determined to be most accurate for the correct identification of species as either $P$. falciparum ( $98 \%$ of cases) or "others" (100\% of cases).

\section{Interference by non-malarial factors}

To exclude potential interference by non-malarial factors, malaria-negative samples with isolated low haemoglobin levels $(n=22)$, isolated low platelet counts $(n=24)$, haemoglobinopathies $(n=23$; thalassaemia $n=14$ and sickle cell disease $n=9$ ) and raised reticulocyte counts $(n=25)$ were analysed. Examples of M scattergrams generated from samples of patients with thalassaemia, sickle cell disease and reticulocytosis are shown in Additional file 1: Fig. S5. None of these samples generated a false positive result. However, an "abnormal MI-RBC scattergram" flag occurred in 30\% (30/101) and 36\% (37/103) of samples analysed in WB and LM modes, respectively, making these results indeterminate. Haemoglobinopathy 


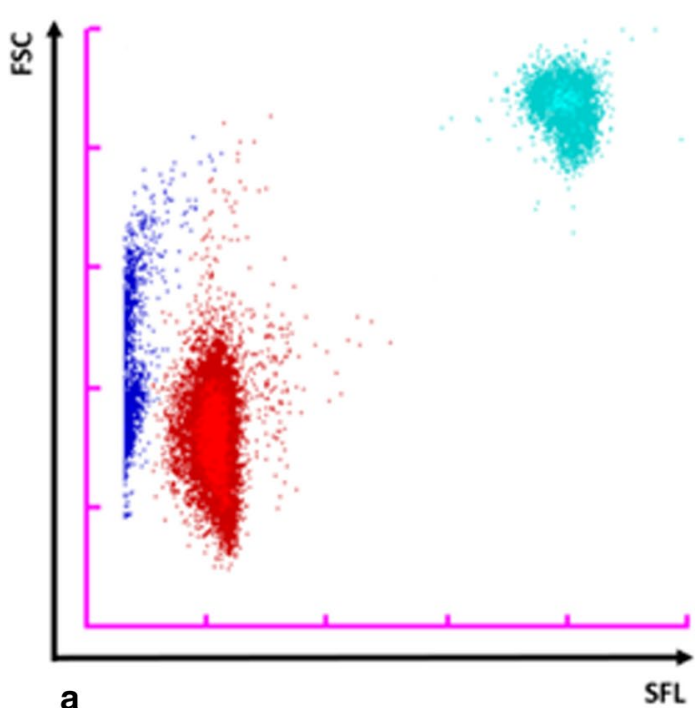

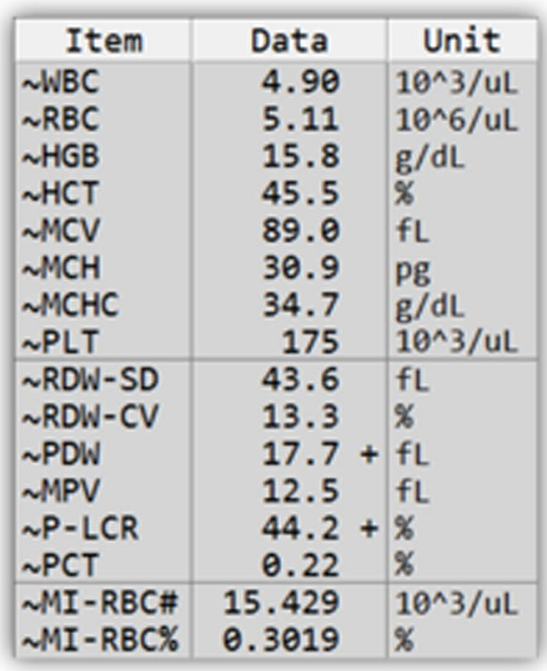

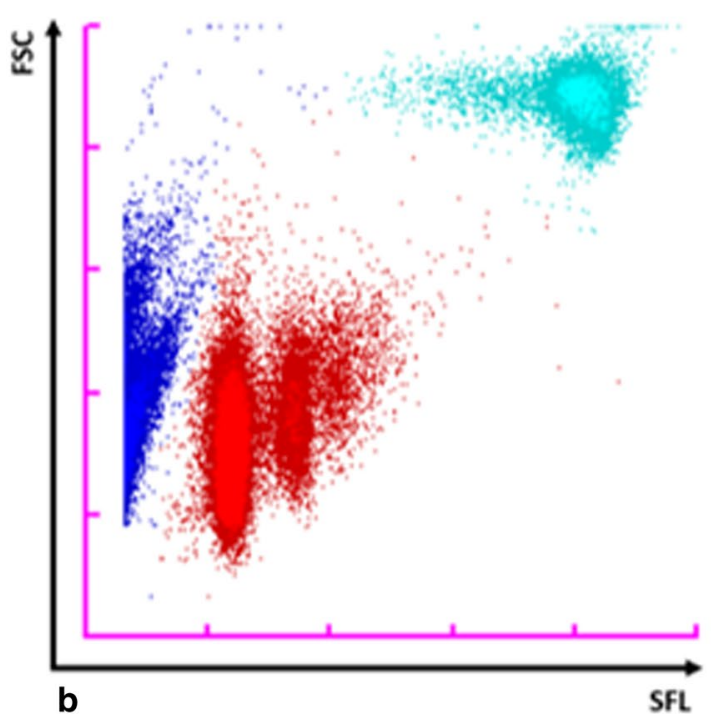

\begin{tabular}{|l|r|l|}
\hline \multicolumn{1}{|c|}{ Item } & Data & \multicolumn{1}{|c|}{ Unit } \\
\hline$\sim$ WBC & 10.34 & $10^{\wedge} 3 / \mathrm{uL}$ \\
$\sim \mathrm{RBC}$ & 3.31 & $10^{\wedge} 6 / \mathrm{uL}$ \\
$\sim \mathrm{HGB}$ & 9.2 & $\mathrm{~g} / \mathrm{dL}$ \\
$\sim \mathrm{HCT}$ & 28.0 & $\%$ \\
$\sim \mathrm{MCV}$ & 84.6 & $\mathrm{fL}$ \\
$\sim \mathrm{MCH}$ & 27.8 & $\mathrm{Pg}$ \\
$\sim$ MCHC & 32.9 & $\mathrm{~g} / \mathrm{dL}$ \\
$\sim \mathrm{PLT}$ & 94 & $10^{\wedge} 3 / \mathrm{uL}$ \\
\hline$\sim \mathrm{RDW}-\mathrm{SD}$ & 43.0 & $\mathrm{fL}$ \\
$\sim \mathrm{RDW}-\mathrm{CV}$ & 13.9 & $\%$ \\
$\sim \mathrm{PDW}$ & 21.4 & $\mathrm{fL}$ \\
$\sim \mathrm{MPV}$ & $13.4 *$ & $\mathrm{fL}$ \\
$\sim \mathrm{P}-\mathrm{LCR}$ & $51.6 *$ & $\%$ \\
$\sim \mathrm{PCT}$ & $0.13 *$ & $\%$ \\
\hline$\sim \mathrm{MI}-\mathrm{RBC}$ & 53.907 & $10^{\wedge} 3 / \mathrm{uL}$ \\
$\sim \mathrm{MI}-\mathrm{RBC}$ & 1.6286 & $\%$ \\
\hline
\end{tabular}

Fig. 2 XN-30 M scattergrams illustrating the detection of red blood cells infected with P. falciparum malaria parasites (MI-RBCs) using WB mode. The corresponding FBC and quantitative MI-RBC parameters (MI-RBC\# and MI-RBC\%) are shown to the right of the scattergrams. SFL: side fluorescence light; FSC: forward scattered light; blue dots: non-infected RBCs, platelets and debris; red dots: MI-RBCs; turquoise dots: white blood cells; "+": value exceeds upper limit; " -": value exceeds the lower limit; *value has low reliability. a Sample with a low parasitaemia of 0.3019\%. b Sample with a higher parasitaemia of $1.6286 \%$

and reticulocytosis samples were most affected. Notably, during the analysis of samples with raised reticulocyte counts, a single case with numerous Howell-Jolly body-containing RBCs (HJB-RBC) was identified. For this sample, the analyser software triggered an "abnormal MI-RBC scattergram" flag and suppressed the MI-RBC values (Fig. 4). The result was ultimately reported by the $\mathrm{XN}-30$ to be indeterminate, and not falsely positive for malaria, despite the presence of stainable intra-erythrocytic nucleic acids.

\section{Analytical evaluation of the Sysmex XN-30 Detection limits}

The LoB $(\mathrm{n}=75)$, LoD $(\mathrm{n}=4)$ and $\operatorname{LoQ}(\mathrm{n}=3)$ are shown in Table 1 . These findings represent values which are much lower compared to field microscopy, for which the reported LoD is $>100$ parasites $/ \mu \mathrm{L}$ [34]. The analyser software is designed to suppress any result below the LoQ. 


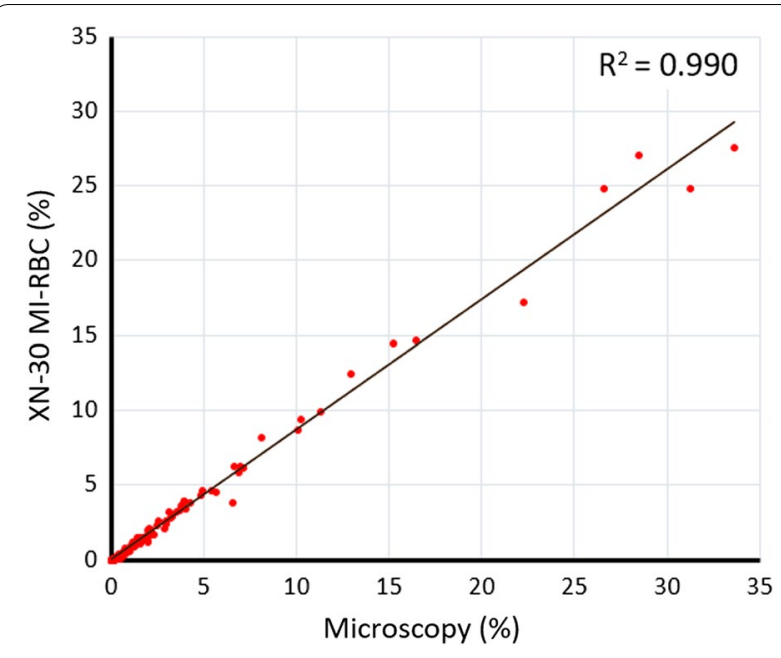

Fig. 3 Correlation between P. falciparum parasitaemia obtained from the XN-30 (MI-RBC\%) and expert microscopy. Samples were analysed in WB mode. $R^{2}$ indicates the coefficient of determination. The diagonal line represents the regression line

\section{Carryover}

Carryover was observed in the LM mode in a single instance whereby an MI-RBC\# of 21 parasites/ $\mu \mathrm{L}$ (LoQ: 20 parasites $/ \mu \mathrm{L}$; Table 1 ) was reported for a blank measurement, which was immediately preceded by a sample with $5.9 \%$ parasitaemia. The manufacturer has since upgraded the $\mathrm{XN}-30$ software to automatically trigger an additional rinse cycle after processing a high $(>5 \%)$ parasitaemia sample, to eliminate the risk of any clinically significant carryover. This is currently under evaluation in the Johannesburg research laboratory. Carryover was not observed in the WB or PD modes.

\section{Precision}

The results of within-run precision analysis for all modes are shown in Additional file 2: Table S3. The CVs of MI$\mathrm{RBC} \#$ and MI-RBC\% in WB, LM and PD modes ranged from $0.7-3.5 \%, 0.4-6.7 \%$ and $0.4-4.7 \%$ respectively.

\section{Stability}

The MI-RBC\# and MI-RBC \% parameters were stable when samples were stored at room temperature (Fig. 5) and $4-8{ }^{\circ} \mathrm{C}$ (Additional file 1: Fig. S6) for at least $60 \mathrm{~h}$ after specimen collection from the patient. The MI-RBC\# for the room temperature samples ranged from approximately $4-136 \times 10^{3} / \mu \mathrm{L}$.

\section{Detection of gametocytes}

The XN-30 generated distinct $M$ scattergrams when in vitro cultured $P$. falciparum gametocytes were analysed, depicted by discrete clusters (Fig. 6). These findings demonstrate the ability of the analyser to detect the transmissible sexual forms of the parasite. Clinical samples with gametocytes were not available for analysis.

\section{Discussion}

Several groups have described the potential of automated analysers to diagnose malaria based on atypical light scatter patterns due to the presence of haemozoin within phagocytic leukocytes $[17,18,21]$. However, these are indirect methods of detecting the parasite and depend on the host immune response to the pathogen, thus the diagnosis of malaria would still require verification by microscopy. Furthermore, haemozoin can persist in circulating monocytes [35]. Thus, the automated detection of haemozoin suffers the same limitation as RDTs, where positive results may be generated after the patient has been treated with anti-malarial agents, and the parasites have been cleared. Detection of intra-erythrocytic haemozoin has also been reported, but it has low sensitivity for diagnosing $P$. falciparum malaria [23].

This report is the first description of the practical and clinical utility of automated, direct detection of malaria parasites in RBCs from infected patients using the Sysmex XN-30 analyser. It demonstrated excellent sensitivity and specificity for $P$. falciparum parasites, which it quantitated accurately and reproducibly. The parasitaemia results of the $\mathrm{XN}-30$ correlated with the current malaria clinical diagnostic gold standard thereby making it as good as expert microscopy for the diagnosis of malaria in a clinical setting.

This novel technology expands the repertoire of malaria diagnostic tools and provides several advantages, which make it applicable to a wide range of clinical scenarios. The $\mathrm{XN}-30$ detects different life cycle stages of the parasite, based on specific $M$ scattergrams, enabling malaria infections to be categorized into either P. falciparum or "others". Two samples of $P$. ovale were classified as "others" and all P. falciparum samples were correctly identified. No mixed infections were observed. Since $P$. falciparum is responsible for $>90 \%$ of malaria deaths it is important that this species is correctly identified. In the few cases where both flags were present, this does not pose a diagnostic problem. Clinically relevant species identification is an invaluable property that makes the $\mathrm{XN}$-30 suitable for application on a global scale, particularly in African countries where P. falciparum is most prevalent, but also in non-African countries, where other species such as $P$. vivax predominate. Furthermore, it may be of particular value in the detection of $P$. knowlesi for which RDTs are not reliable and microscopy skills may be limited due to the more rural distribution of such cases [15]. 


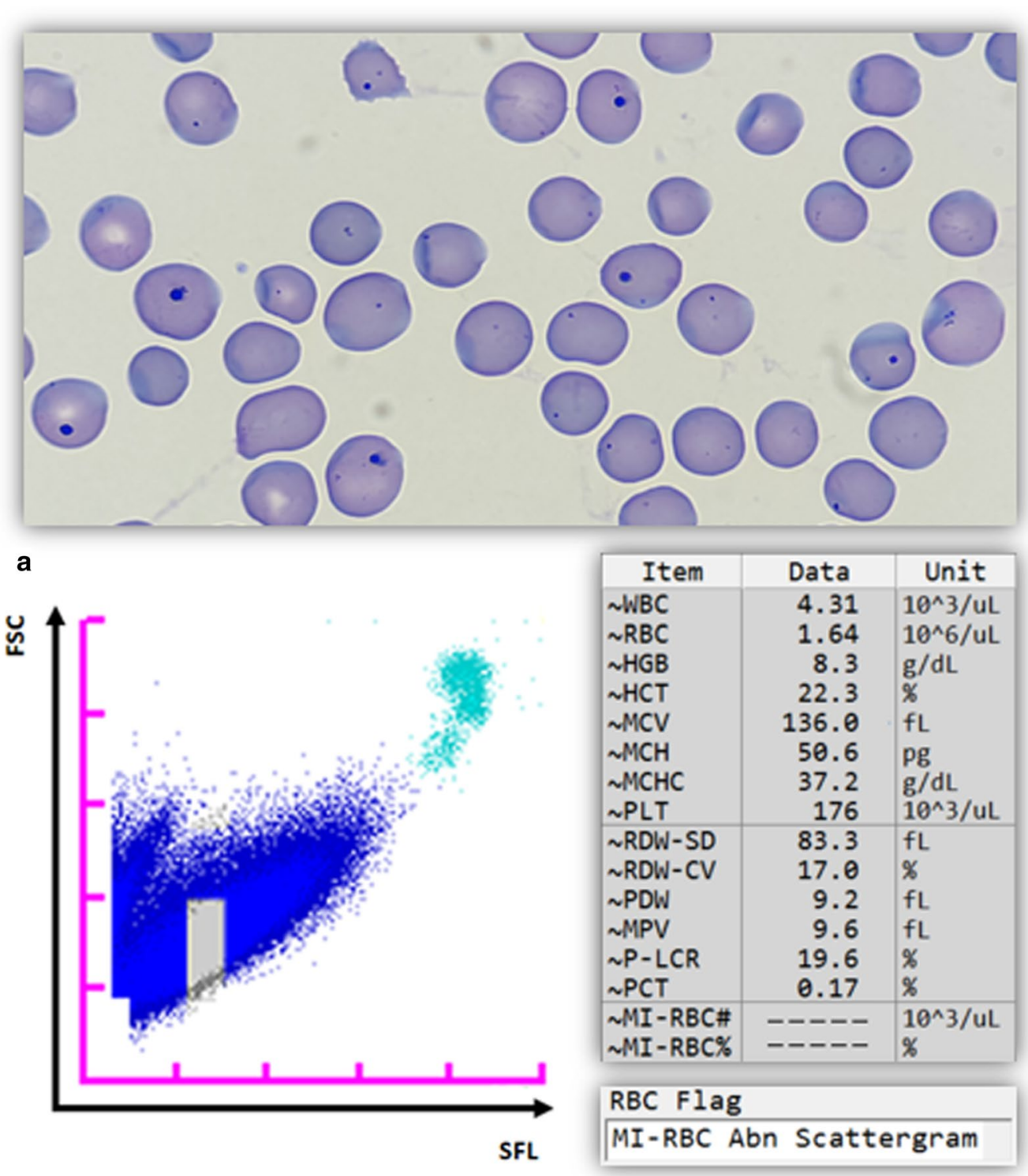

b

C

Fig. 4 Lack of interference by Howell-Jolly bodies with the quantitative MI-RBC parameters. a Peripheral blood smear of the sample with numerous Howell-Jolly body-containing RBCs. b The sample was analysed on the XN-30 in WB mode and the M scattergram shows a large blue cluster with a grey area, reflecting the region where MI-RBCs scatter. Turquoise dots: white blood cells. c The MI-RBC parameters (MI-RBC\# and MI-RBC\%) were suppressed and the software triggered an "abnormal MI-RBC scattergram"flag in response to the large blue cluster and the result was reported as indeterminate

Table 1 Limits of blank (LoB), detection (LoD) and quantitation (LoQ) for all modes of the XN-30

\begin{tabular}{llll}
\hline & $\begin{array}{l}\text { Whole blood } \\
\text { (WB) }\end{array}$ & $\begin{array}{l}\text { Low malaria } \\
\text { (LM) }\end{array}$ & $\begin{array}{l}\text { Pre- } \\
\text { dilute } \\
\text { (PD) }\end{array}$ \\
\hline Limit of blank (LoB) & 13 & 12 & 16 \\
Limit of detection (LoD) & 22 & 20 & 36 \\
Limit of quantitation (LoQ) & 30 & 20 & 40 \\
\hline
\end{tabular}

Values reported as MI-RBC\#
The XN-30 is user-friendly, and it takes approximately $1 \mathrm{~min}$ to analyse each sample, including specimen aspiration, performance of measurements and reporting of results, which requires minimal technical input or expertise. Likewise, the maintenance requirements, being comprised only of a daily analyser shutdown, are minimal. Furthermore, in the event of any other "as needed" maintenance, the user will be prompted by an on screen "pop-up" message which includes a step by 


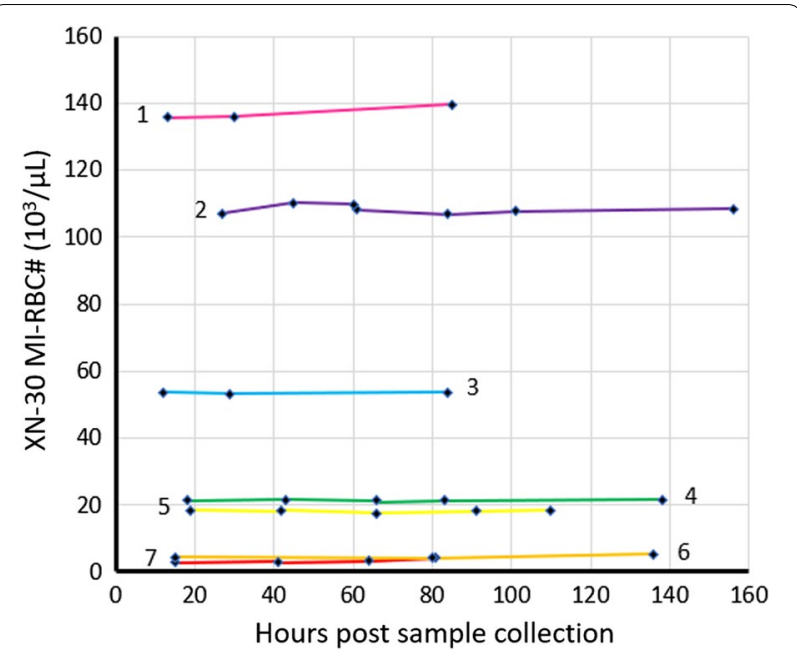

Fig. 5 Stability of MI-RBC parameters (MI-RBC\#) measured in WB mode on the $\mathrm{XN}$-30. Seven samples with P. falciparum parasitaemia ranging from low to high $\left(4-136 \times 10^{3} / \mu \mathrm{L}\right)$, were stored at room temperature and analysed at various time intervals. The first measurement was performed within $24 \mathrm{~h}$ of sample collection from the patient and thus the initial point is different for each sample

step guide, thereby making all maintenance very simple and easy to execute. In fact, the maintenance of the $\mathrm{XN}-30$ is far simpler than that of the smaller Sysmex haematology analysers, such as the KX-21N, for which there is a large footprint in decentralized laboratories throughout Africa and Asia. Overall, its rapid processing time and objective, automated analysis facilitates high throughput of samples, highlighting the suitability of the $\mathrm{XN}-30$ to malaria endemic regions.

In addition, the MI-RBC parameters are robust and unaffected by room temperature storage of samples, thereby enabling specimens collected even in remote locations to be transported at ambient temperature for analysis.

The quantitative $\mathrm{XN}-30$ technology enables an accurate number of infected RBCs and percentage parasitaemia to be reported, thereby facilitating the monitoring of the decrease in parasite load and thus therapeutic efficacy once treatment with anti-malarial drugs has been initiated. Such properties may allow for early identification of drug resistance to the current front line artemisininbased combination therapy (ACT), and thus yield valuable data on the spread of artemisinin resistance, which is alarmingly prevalent in the Greater Mekong sub-region [36, 37].

Another important advantage of the $\mathrm{XN}-30$ is that, in addition to the unique property of directly identifying parasites in RBCs, it generates a conventional FBC with each result, which none of the other malaria diagnostic tools offer. This provides clinicians with baseline blood counts and enables them to evaluate the haematological response of the patient during treatment. This feature will also be beneficial in clinical research applications, such as population studies and clinical trials to test new anti-malarial drugs or vaccines. This has recently been exploited in a study of a mouse model of malaria whereby the pharmacokinetics of drugs, as well as therapy-related safety profiles, were investigated on the $\mathrm{XN}-30$ [33]. An
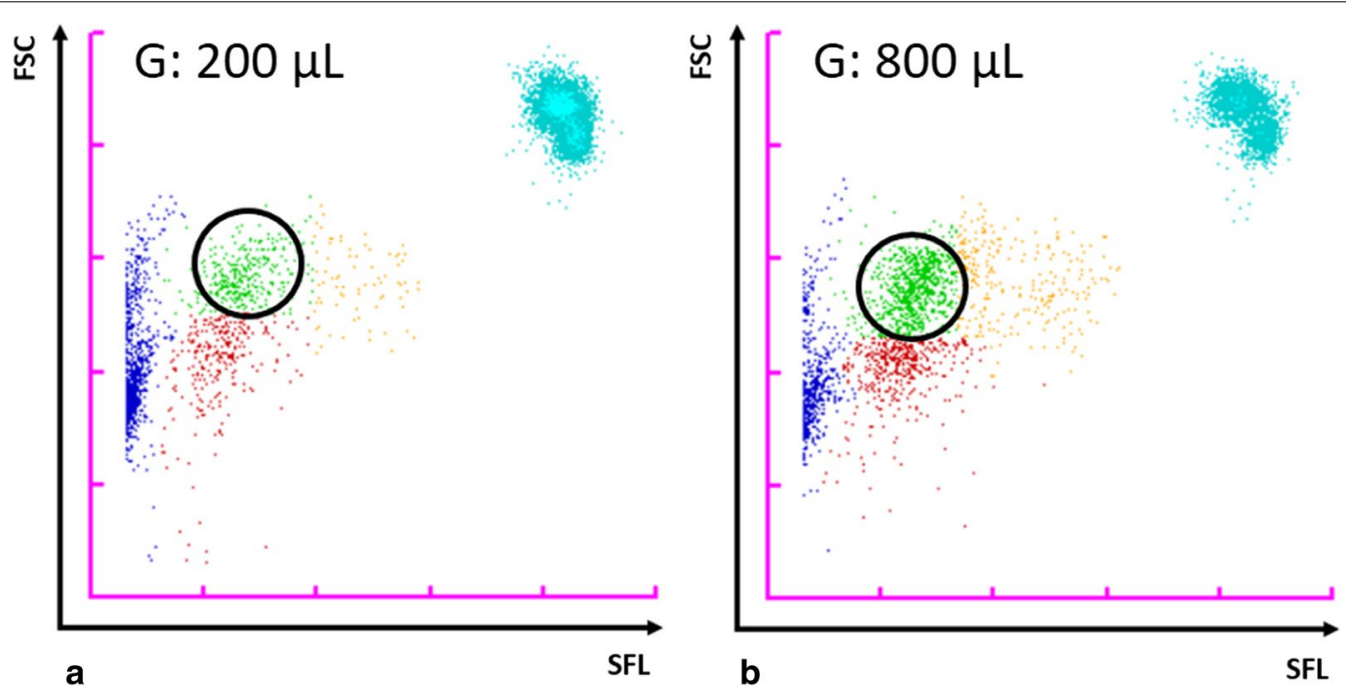

Fig. $6 \mathrm{XN}-30 \mathrm{M}$ scattergrams illustrating the detection of $P$. falciparum gametocytes. Malaria-negative whole blood (1 mL) spiked with $200 \mu \mathrm{L}(\mathbf{a})$ and $800 \mu \mathrm{L}$ (b) of purified P. falciparum gametocytes. SFL: side fluorescence light; FSC: forward scattered light; circled green dots: gametocytes. These samples were analysed in WB mode 
additional significant benefit of the concurrent detection of malaria parasites and generation of a $\mathrm{FBC}$ is that it may prove useful in identifying unsuspected cases of malaria. In scenarios where there is a low index of suspicion for the disease, a FBC is typically requested as part of an initial diagnostic blood panel in a febrile patient, and the $\mathrm{XN}-30$ will automatically flag the sample if malaria parasites are present. All other diagnostic tools require a directed request from a health care professional to test for malaria. Since prompt diagnosis and initiation of appropriate treatment is vital in preventing complications of malaria and potential death of the patient, the $\mathrm{XN}-30$ may have an impact in identifying infection in countries in the pre-elimination phase and in tourists returning home to non-malaria endemic countries.

Transfusion-transmitted malaria (TTM) is a wellrecognized entity and is fast emerging as a major public health concern, particularly in malaria-endemic African countries. The prevalence of $P$. falciparum carriage can range from $2 \%$ to more than $55 \%$ among healthy blood donors [38-40]. Current screening protocols in these regions pose a diagnostic challenge as the commonly used methods are not sufficiently sensitive [41]. The $\mathrm{XN}-30$ may, therefore, serve as the ideal donor screening tool in blood banks of malaria-endemic regions. The simultaneous haematological evaluation may also provide an indication of donor health status and thus such a strategy may eliminate the risk of TTM and improve the overall quality of blood products obtained.

A final important benefit of the XN-30 is that it can detect gametocytes as demonstrated here and by a Japanese group [24] using in vitro P. falciparum culture samples. Gametocytes are critical for parasite survival since they are transmitted to the mosquito vector where they initiate the sexual phase of the parasite life cycle, which results in the production of sporozoites rendering the mosquito infectious and allowing the spread of the disease. The $\mathrm{XN}-30$ may be harnessed to identify asymptomatic carriers who can be treated to prevent transmission of gametocytes and therefore disrupt the parasite life cycle. This strategy is a key component of global initiatives to eliminate the disease. Automated detection of gametocytes may also be useful for further research on gametocytogenesis in patients/asymptomatic carriers and for transmission-blocking drug discovery.

A limitation of the current $\mathrm{XN}-30$ is the number of indeterminate results obtained due to the reported "abnormal MI-RBC scattergram" flags. Despite this only occurring in malaria-negative samples, with no false positive results being generated, it may potentially impact on routine workflow, as affected samples would require microscopic review. Whilst the exact cause of the "abnormal MI-RBC scattergram" flag in the malaria-negative samples is currently unknown, the disproportionate occurrence in specimens obtained from patients with reticulocytosis, suggests that it may be related to high red cell turnover. This is currently under investigation by the manufacturer.

\section{Conclusions}

Timely detection of malaria is life-saving and whilst most health care professionals are trained to recognize the infection, clinical presentation is often non-specific, and diagnosis may initially be overlooked. The XN-30 analyser is an ideal modality for the precise recognition and automated enumeration of malaria parasitaemia. It rapidly detects the actual parasite and not any by-product, such as antigens, phagocytosed parasites or haemozoin, making it more suitable for malaria detection compared to RDTs and other indirect automated methods. Concurrent measurement of haematological parameters is a unique feature that provides valuable data for clinical correlation. Whilst it may not immediately replace microscopy for confirmation of clinical malaria infection, the $\mathrm{XN}-30$ should certainly be considered for utilization as an adjunctive diagnostic tool, because it can facilitate the detection of unsuspected cases. Overall, this technological advance has the potential to significantly impact on malaria diagnostic approaches, therapeutic monitoring and efficacy, anti-malarial drug discovery and clinical trials.

\section{Additional files}

Additional file 1: Fig. S1. XN-30 software improvements. a. In the prototype software, the M scattergram plot area was divided into 3 regions classifying signals as WBCs (cluster a), non-infected RBCs, platelets and debris (cluster b) and MI-RBCs (cluster c). In brief, the gating strategy sequence was to first identify cluster a and then cluster $b$. In the prototype, all remaining signals were then assigned to cluster c. Upon further investigation, it was identified that the specified MI-RBC area (cluster $\mathrm{c}$ ) was too broad, giving rise to false positive MI-RBC results. $\mathbf{b}$. The software was subsequently improved for the $\mathrm{XN}-30$ by narrowing the $\mathrm{MI}-\mathrm{RBC}$ area (cluster $\mathrm{c}$ ) and incorporating the recognition of one or more distinct clusters (ring forms, gametocytes, trophozoites/schizonts) within an appropriate shape and position as a prerequisite for generating an MI-RBC result. Fig. S2. XN-30 malaria classification algorithm. The left side illustrates the algorithm flow if a malaria cluster is not recognized. In such cases, if the $\mathrm{MI}-\mathrm{RBC} \#$ is $<\mathrm{LOQ}$, the sample is reported as malaria negative (MI-RBC green box). However, if the MI-RBC\# is $\geq$ LoQ but signals are detected in the absence of appropriate clustering, or an incorrect cluster shape is generated within the MI-RBC area, then the result is suppressed and an MI-RBC abnormal scattergram flag is generated. An indeterminate result is reported (MI-RBC grey box). An example is presented in M scattergram (a). The right side illustrates the algorithm flow if a malaria cluster is recognized. In such cases, if the MI-RBC\# is $<$ LoQ, the sample is reported as malaria negative (MI-RBC green box). However, if the MI-RBC\# is $\geq$ LoQ the sample is reported as malaria positive ( $M I-R B C$ red box). An example is presented in M scattergram (b). Fig. S3. XN-30 M scattergrams, MI-RBC values and the species RBC flags for patient samples infected with a $P$. falciparum (P. f) and $\mathbf{b}$ P. ovale (others). Measurements were performed 
in WB mode. P. ovale parasites were confirmed microscopically. SFL: side fluorescence light; FSC: forward scattered light; blue dots: non-infected RBCs, platelets and debris; red dots: MI-RBCs; green and orange dots: different $P$. ovale life cycle forms; turquoise dots: white blood cells. Fig. S4. Correlation between P. falciparum parasitaemia obtained from the XN-30 (MI-RBC\%) and expert microscopy. Samples were analysed in a LM mode and $\mathbf{b}$ PD mode. $R^{2}$ indicates the coefficient of determination. The diagonal lines represent the regression lines. Fig. S5. XN-30 M scattergrams of malaria-negative patient samples with $\mathbf{a}$ thalassaemia, $\mathbf{b}$ sickle cell disease and $\mathbf{c}$ reticulocytosis of $13.86 \%$. The grey area within the large blue cluster reflects the region where MI-RBCs scatter, but the software correctly triggered an "abnormal MI-RBC scattergram". Measurements were performed in WB mode. SFL: side fluorescence light; FSC: forward scattered light; blue dots: non-infected RBCs, platelets and debris; turquoise dots: white blood cells. Fig. S6. Stability of MI-RBC parameters (MI-RBC\#) at $4-8{ }^{\circ} \mathrm{C}$ measured in WB mode on the $\mathrm{XN}-30$. Four samples with P. falciparum parasitaemia ranging from a high $\left(21-107 \times 10^{3} / \mu \mathrm{L}\right)$, to $\mathbf{b}$ low $\left(3-4.5 \times 10^{3} / \mu \mathrm{L}\right)$, were stored at $4-8{ }^{\circ} \mathrm{C}$ and analysed at various time intervals. The first measurement was performed within $24 \mathrm{~h}$ of sample collection from the patient and thus the initial point is different for each sample.

Additional file 2: Table S1. WB mode data and M scattergrams. Table S2. LM mode data and M scattergrams. Table S3. All modes precision data.

\section{Abbreviations}

WHO: World Health Organization; RDT: rapid diagnostic test; PCR: polymerase chain reaction; LAMP: loop-mediated isothermal amplification; HRP2: histidine-rich protein 2; FBC: full blood count; WBC: white blood cell; RBC: red blood cell; DC: direct current; MI-RBC: malaria-infected red blood cell; MI-RBC\#: malaria-infected red blood cell count; MI-RBC\%: malaria-infected red blood cell percentage; SLS: sodium lauryl sulphate; SFL: side fluorescence light; FSC: forward scattered light; ACAS: adaptive cluster analysis system; RSA: Republic of South Africa; WB: whole blood; LM: low malaria; PD: pre-dilute; NICD: National Institute for Communicable Diseases; HIV: human immunodeficiency virus; LoB: limit of blank; LoD: limit of detection; LoQ: limit of quantitation; SD: standard deviation; CV: coefficient of variation; Cl: confidence interval; HJB-RBC: Howell-Jolly body-containing red blood cell; ACT: artemisinin-based combination therapy; TTM: transfusion-transmitted malaria.

\section{Authors' contributions}

EP conducted the $\mathrm{XN}-30$ study and compiled the figures and tables. EP and TLC performed microscopy, evaluated the data, drafted and critically revised the manuscript. BCB and SK performed the prototype study in RSA and India, respectively. ML performed microscopy for the $\mathrm{XN}-30$ and prototype study in RSA and provided technical assistance. All authors read and approved the final manuscript.

\section{Author details}

${ }^{1}$ Wits Research Institute for Malaria, Department of Molecular Medicine and Haematology, School of Pathology, Faculty of Health Sciences, University of the Witwatersrand and National Health Laboratory Service, Johannesburg, South Africa. ${ }^{2}$ Hematology Department, P. D. Hinduja National Hospital \& Medical Research Centre, Mumbai, India. ${ }^{3}$ Department of Microbiology, Chris Hani Baragwanath Academic Hospital, National Health Laboratory Service, Johannesburg, South Africa.

\section{Acknowledgements}

We thank the following individuals who contributed to the study: Prof. Jeannette Wadula and Prof. Adriano Duse for access to residual blood samples; Prof. John Frean and Bhavani Moodley for expert advice in identifying P. ovale samples; Desiree du Plessis who performed PCR for the XN-30 study; the medical technologists in the routine laboratories; Dr. Marion Münster, Dr. Jarob Saker, Ndwakulu Nemuthengame and Tiaan van den Berg for Sysmex technical support.

\section{Competing interests}

The study was funded by Sysmex Corporation, Kobe, Japan and guidelines for the analytical performance were provided by the manufacturer. The authors interpreted the results objectively and had access to all the raw data. The discussion of the advantages of the technology was based on the quality of the data and the authors' experience in the malaria field and was not influenced by the manufacturer.

\section{Availability of data and materials}

All data generated and analysed on the Sysmex $\mathrm{XN}-30$ during this study are included in this published article and its supplementary information files. The datasets used and analysed during the prototype study are available from the corresponding author on reasonable request.

\section{Consent for publication}

Not applicable.

\section{Ethics approval and consent to participate}

This study was approved by the Human Research Ethics Committee of the University of the Witwatersrand (M140995 and M160549) in RSA and by the National Health and Education Society (872-14-SK/KS) in India.

\section{Funding}

The study was funded by Sysmex Corporation, Kobe, Japan.

\section{Publisher's Note}

Springer Nature remains neutral with regard to jurisdictional claims in published maps and institutional affiliations.

Received: 28 November 2018 Accepted: 16 January 2019

Published online: 22 January 2019

\section{References}

1. WHO. World malaria report 2018. Geneva: World Health Organization; 2018. http://www.who.int/malaria/publications/world-malaria-repor t-2018/en/. Accessed 22 Nov 2018.

2. Chotivanich K, Silamut K, Day N. Laboratory diagnosis of malaria infection - a short review of methods. N Z J Med Lab Sci. 2007:61:4-7.

3. WHO. Guidelines for the treatment of malaria. 3rd edition. Geneva: World Health Organization; 2015. http://www.who.int/malaria/publications/ atoz/9789241549127/en/. Accessed 14 Nov 2018.

4. Coleman RE, Sattabongkot J, Promstaporm S, Maneechai N, Tippayachai B, Kengluecha A, et al. Comparison of PCR and microscopy for the detection of asymptomatic malaria in a Plasmodium falciparum/vivax endemic area in Thailand. Malar J. 2006:5:121.

5. Hopkins H, Gonzalez IJ, Polley SD, Angutoko P, Ategeka J, Asiimwe C, et al. Highly sensitive detection of malaria parasitemia in a malaria-endemic setting: performance of a new loop-mediated isothermal amplification kit in a remote clinic in Uganda. J Infect Dis. 2013;208:645-52.

6. WHO. New perspectives: malaria diagnosis — report of a joint WHO/ USAID informal consultation, 25-27 October 1999. Geneva: World Health Organization; 2000. http://www.who.int/tdr/publications/documents/ malaria-diagnosis.pdf. Accessed 26 Oct 2018.

7. Abba K, Kirkham AJ, Olliaro PL, Deeks JJ, Donegan S, Garner P, et al. Rapid diagnostic tests for diagnosing uncomplicated non-falciparum or Plasmodium vivax malaria in endemic countries. Cochrane Database Syst Rev. 2014;12:CD011431.

8. Bain BJ. Blood cells: a practical guide. 5th ed. London: Academic; 1995. p. $148-68$.

9. Sori G, Zewdie O, Tadele G, Samuel A. External quality assessment of malaria microscopy diagnosis in selected health facilities in Western Oromia, Ethiopia. Malar J. 2018;17:233.

10. Eshel Y, Houri-Yafin A, Benkuzari H, Lezmy N, Soni M, Charles M, et al. Evaluation of the Parasight platform for malaria diagnosis. J Clin Microbiol. 2017;55:768-75.

11. Odaga J, Sinclair D, Lokong JA, Donegan S, Hopkins H, Garner P. Rapid diagnostic tests versus clinical diagnosis for managing people with fever in malaria endemic settings. Cochrane Database Syst Rev. 2014;4:CD008998.

12. Dalrymple U, Arambepola R, Gething PW, Cameron E. How long do rapid diagnostic tests remain positive after anti-malarial treatment? Malar J. 2018;17:228. 
13. Grobusch MP, Alpermann U, Schwenke S, JelinekT, Warhurst DC. Falsepositive rapid tests for malaria in patients with rheumatoid factor. Lancet. 1999;353:297.

14. WHO. World malaria report 2017. Geneva: World Health Organization; 2017. http://www.who.int/malaria/publications/world-malaria-repor t-2017/en/. Accessed 6 Nov 2018.

15. Singh B, Daneshvar C. Human infections and detection of Plasmodium knowlesi. Clin Microbiol Rev. 2013;26:165-84.

16. Singh A, Narang V, Sood N, Garg B, Gupta VK. Malaria diagnosis using automated analysers: a boon for hematopathologists in endemic areas. J Clin Diagn Res. 2015;9:EC05-8.

17. Mendelow BV, Lyons C, Nhlangothi P, Tana M, Munster M, Wypkema E, et al. Automated malaria detection by depolarization of laser light. Br J Haematol. 1999;104:499-503.

18. Briggs C, Da Costa A, Freeman L, Aucamp I, Ngubeni B, Machin SJ. Development of an automated malaria discriminant factor using VCS technology. Am J Clin Pathol. 2006;126:691-8.

19. Mohapatra S, Samantaray JC, Arulselvi S, Panda J, Munot K, Saxena R. Automated detection of malaria with haematology analyzer Sysmex XE-2100. Indian J Med Sci. 2011;65:26-31.

20. Sunilkumar K, Naik P. Usefulness of automated hematology analyzer Sysmex XN 1000 in detection of malaria. Indian J Path Onc. 2016;3:658-61.

21. Hänscheid T, Valadas E, Grobusch MP. Automated malaria diagnosis using pigment detection. Parasitol Today. 2000;16:549-51.

22. Kramer B, Grobusch MP, Suttorp N, Neukammer J, Rinneberg H. Relative frequency of malaria pigment-carrying monocytes of nonimmune and semi-immune patients from flow cytometric depolarized side scatter. Cytometry. 2001;45:133-40.

23. Rebelo M, Shapiro HM, Amaral T, Melo-Cristino J, Hänscheid T. Haemozoin detection in infected erythrocytes for Plasmodium falciparum malaria diagnosis-prospects and limitations. Acta Trop. 2012;123:58-61.

24. Tougan T, Suzuki Y, Itagaki S, Izuka M, Toya Y, Uchihashi K, et al. An automated haematology analyzer XN-30 distinguishes developmental stages of falciparum malaria parasite cultured in vitro. Malar J. 2018;17:59.

25. Venter JC, Adams MD, Myers EW, Li PW, Mural RJ, Sutton GG, et al. The sequence of the human genome. Science. 2001;291:1304-51.

26. Carlton JM, Adams JH, Silva JC, Bidwell SL, Lorenzi H, Caler E, et al. Comparative genomics of the neglected human malaria parasite Plasmodium vivax. Nature. 2008;455:757-63.

27. Crutcher JM, Hoffman SL. Malaria. In: Baron S, editor. Medical microbiology. 4th ed. Galveston: University of Texas medical branch at Galveston; 1996.

28. WHO. Microscopy for the detection, identification and quantification of malaria parasites on stained thick and thin blood films in research settings. Geneva: World Health Organization; 2015. http://www.who.int/ tdr/publications/microscopy_detec_ident_quantif/en/. Accessed 10 Nov 2018.

29. Hang VT, Van Be T, Tran PN, Thanh LT, Van Hien L, O'Brien E, et al. Screening donor blood for malaria by polymerase chain reaction. Trans $\mathrm{R}$ Soc Trop Med Hyg. 1995;89:44-7.

30. Armbruster DA, Pry T. Limit of blank, limit of detection and limit of quantitation. Clin Biochem Rev. 2008;29(Suppl 1):S49-52.

31. Reader J, Botha M, Theron A, Lauterbach SB, Rossouw C, Engelbrecht $D$, et al. Nowhere to hide: interrogating different metabolic parameters of Plasmodium falciparum gametocytes in a transmission blocking drug discovery pipeline towards malaria elimination. Malar J. 2015;14:213.

32. Pillay $E$, Litshie $M$, Coetzer TL. A new era in malaria diagnosis and surveillance using an automated analyzer. Blood. 2017;130(Suppl 1):2097.

33. Tougan T, Suzuki Y, Izuka M, Aono K, Okazaki T, Toya Y, et al. Application of the automated haematology analyzer XN-30 in an experimental rodent model of malaria. Malar J. 2018;17:165.

34. WHO. Evidence review group on low-density malaria infections. Geneva: World Health Organization; 2017. http://www.who.int/malaria/mpac/ mpac-oct2017-erg-malaria-low-density-infections-session2.pdf. Accessed 20 Oct 2018.

35. Boura M, Frita R, Gois A, Carvalho T, Hänscheid T. The hemozoin conundrum: is malaria pigment immune-activating, inhibiting, or simply a bystander? Trends Parasitol. 2013;29:469-76.

36. WHO. Emergency response to artemisinin resistance in the Greater Mekong subregion. Geneva: World Health Organization; 2013. http:// www.who.int/malaria/publications/atoz/9789241505321/en/. Accessed 28 Oct 2018.

37. Imwong M, Suwannasin K, Kunasol C, Sutawong K, Mayxay M, Rekol H, et al. The spread of artemisinin-resistant Plasmodium falciparum in the Greater Mekong subregion: a molecular epidemiology observational study. Lancet Infect Dis. 2017;17:491-7.

38. Owusu-Ofori A, Gadzo D, Bates I. Transfusion-transmitted malaria: donor prevalence of parasitaemia and a survey of healthcare workers knowledge and practices in a district hospital in Ghana. Malar J. 2016;15:234.

39. Kouriba B, Diarra AB, Douyon I, Diabate DT, Kamissoko F, Guitteye H, et al. Plasmodium falciparum malaria prevalence among blood donors in Bamako, Mali. Transfus Clin Biol. 2017;24:62-7.

40. Nansseu JRN, Noubiap JJN, Ndoula ST, Zeh AFM, Monamele CG. What is the best strategy for the prevention of transfusion-transmitted malaria in sub-Saharan African countries where malaria is endemic? Malar J. 2013;12:465

41. Owusu-Ofori AK, Betson M, Parry CM, Stothard JR, Bates I. Transfusiontransmitted malaria in Ghana. Clin Infect Dis. 2013;56:1735-41.
Ready to submit your research? Choose BMC and benefit from:

- fast, convenient online submission

- thorough peer review by experienced researchers in your field

- rapid publication on acceptance

- support for research data, including large and complex data types

- gold Open Access which fosters wider collaboration and increased citations

- maximum visibility for your research: over $100 \mathrm{M}$ website views per year

At BMC, research is always in progress.

Learn more biomedcentral.com/submissions 